\title{
Estimación del riesgo en edificaciones por deslizamientos causados por lluvias y sismos en la ciudad de Medellín, empleando herramientas de la Geomática
}

\author{
Johnny Alexander Vega Gutiérrez*
}

Recibido el 5 de diciembre de 2015; aceptado el 2 de septiembre de 2016

\begin{abstract}
This article presents a risk estimation in buildings by landslides triggered by earthquakes and rainfall in the city of Medellin using a Geographic Information System (GIS). For this, a computer model that considers topographic, geological, geotechnical and hydrological characteristics of the study area was implemented to determine landslide hazard by evaluating the stability of slopes using pseudo static Newmark Method, including a probabilistic approach technique based on the first order and second moment -FOSM. The approach for the assessment of physical vulnerability of buildings in the study area was conducted through structural fragility indexes that consider the definition of the level of damage to buildings through event trees using a cadastral inventory data of the municipality of Medellin. The routine calculates the probability of a landslide to occur given that an earthquake produces a horizontal ground acceleration (Ah), considering the uncertainty of the geotechnical parameters and the conditions of soil saturation for the effect of previous rainfall in the area, then multiply such values with the structural fragility indexes values in order to estimate the damage indices of buildings, which are associated with the risk when considering the relevant economic variable to the replacement value of such structures from assessed values.
\end{abstract}

Key words: Buildings, earthquake, GIS, landslide, rainfall, risk.

* Programa de Ingeniería Civil, Facultad de Ingeniería, Universidad de Medellín, Colombia, correo electrónico: javega@udem.edu.co 


\section{Resumo}

Este artigo apresenta uma estimação de riscos por deslizamento para edificações causados por chuvas e sismos na cidade de Medellín, empregando uma plataforma de Sistemas de Informação Geográfica (SIG). Para isso se implementou um modelo que considera as propriedades e os aspectos topográficos, geológicos, geotécnicos e hidrológicos da zona em estudo. O modelo de cálculo usado para avaliar a ameaça por deslizamento, está fundamentado no Método seudo-estático de Newmark para o cálculo de estabilidad de ladeiras e usa um enfoque probabilista baseado no método de primeira ordem e, num segundo momento —o FOSM - para estimar a variação de dos parâmetros geomecânicos do solo. A abordajem para a avaliação da vulnerabilidade das construções da área de estudos se realizou mediante índices de fragilidade estrutural que consideram a definição de níveis de dano através de árvores de decisão fazendo uso da informação cadastral da cidade. O modelo calcula a probabilidade de que ocorra um deslizamento a partir de um sismo que produza uma aceleração (Ah), considerando a incerteza dos parâmetros geotécnicos e as condições de saturação do solo, e multiplica tais valores com os índices de debilidade estrutural para estimar os índices de dano das construções, as quais se associam com o risco a ser considerado e a variável econômica correspondente ao custo de reposição de tais construções.

Palavras chave: edificações, deslizamento, chuvas, risco, SIG, sismo.

\section{Resumen}

Este artículo presenta una estimación del riesgo en edificaciones por deslizamientos causados por lluvias y sismos en la ciudad de Medellín, empleando una plataforma de Sistemas de Información Geográfica (SIG). Para esto se implementó un modelo que considera las propiedades topográficas, geológicas, geotécnicas e hidrológicas de la zona en estudio. El modelo de cálculo usado para la valoración de la amenaza por deslizamiento, está fundamentado en el Método seudoestático de Newmark para el cálculo de estabilidad de laderas y usa un enfoque probabilista el cual se basa en el método de primer orden y segundo momento - FOSM - para estimar la variación de los parámetros geomecánicos del suelo. El abordaje para la valoración de la vulnerabilidad de las construcciones en el área de estudio se realizó mediante índices de fragilidad estructural que consideran la definición de niveles de daño a través de árboles de decisión haciendo uso de la información catastral de la ciudad. El modelo calcula la probabilidad de que ocurra un deslizamiento dado que se presente un sismo que produzca una aceleración (Ah), considerando la incertidumbre de los parámetros geotécnicos y las condiciones de saturación del suelo, y multiplica tales valores con los índices de debilidad estructural para estimar los índices de daño de 
las construcciones, las cuales se asocian con el riesgo al considerar la variable económica correspondiente al costo de reposición de tales construcciones.

Palabras clave: edificaciones, deslizamiento, lluvias, riesgo, SIG, sismo.

\section{Introducción}

Los movimientos en masa, así como las inundaciones son en esencia, los fenómenos naturales que generan los desastres más severos en Colombia. Durante los últimos 40 años, los desastres naturales han ocasionado pérdidas que alcanzan un promedio de pérdidas anuales de US\$177 millones y se han visto afectadas más de un millón de viviendas asociadas a la ocurrencia de diferentes fenómenos, entre ellas, el 5\% por deslizamientos. Entre 1970 y el 2011 fueron destruidas 190 mil viviendas, de las cuales casi 18 mil (9\%) estuvieron asociadas a deslizamientos (Campos et al., 2012).

El Valle de Aburrá (VA) corresponde a una depresión topográfica alargada en zona de montaña, donde se ubica la ciudad de Medellín y sus municipios vecinos, que debido a su relieve, topografía, geología, entre otros, se constituye en una región susceptible para la ocurrencia de procesos geomorfodinámicos, que afectan tanto a la población como a su infraestructura. Dado que en los últimos años se ha evidenciado un incremento en la ocupación de las laderas de manera inadecuada debido a los asentamientos humanos o por obras de infraestructura, los riesgos asociados a los movimientos en masa también han incrementado. Se estima que en el VA el 35\% de los daños a edificaciones y $74 \%$ de las muertes debidas a fenómenos naturales, están asociadas con movimientos en masa (Aristizábal y Gómez, 2007), mientras que a nivel mundial, a tales movimientos se les atribuye el $0.53 \%$ de las muertes debidas a desastres por fenómenos naturales (Chowdhury et al., 2010).

En la evaluación de la amenaza por deslizamientos, muchos trabajos están basados en la aplicación de información espacial asociada a un solo factor detonante, usando tanto teledetección como SIG como ambiente para el procesamiento (Shahabi y Hashim, 2015; Frigerio y Van Westen, 2010; Lan et al., 2004, entre otros), y en general se han enfocado en mapas de susceptibilidad, álgebra de mapas y datos históricos, y muchos de los trabajos realizados adolecen de no considerar el comportamiento de los materiales, los mecanismos de falla, y el tema de la vulnerabilidad es pocamente discutida, y por tanto, la evaluación del riesgo ha sido parcial.

Respecto al tema de la evaluación de la vulnerabilidad, se ha puesto de manifiesto la gran utilidad de la información de un inventario catastral (Botero 2009; Panahi et al., 2011). En general, las evaluaciones de la vulnerabilidad y del riesgo bajo ambiente SIG también ha sido tema de interés en múltiples investigaciones (Van Westen et al., 2008; Van Westen 2013; Martha et al., 2013; Promper et al., 2014; Torkashvand et al., 2014; entre otras). 
En la literatura, varios estudios individuales evidencian el acercamiento al tema de la evaluación cuantitativa del riesgo de deslizamientos (Corominas et al., 2013; Isaza, 2016; Zêzere et al., 2008; Frigerio y Van Westen, 2010; Remondo et al., 2008; entre otros).

En este trabajo se presenta un planteamiento que analiza modelos de base física y probabilística para estimar la amenaza por deslizamiento, y un planteamiento que toma en cuenta índices de fragilidad estructural para las construcciones, calculados por medio del uso de árboles de decisión a partir del inventario catastral del municipio de Medellín. Los factores considerados como detonantes de los deslizamientos fueron sismos y lluvia mediante una aplicación SIG a escala regional para el VA, tomando como zona piloto para la estimación del riesgo, la comuna nororiental de la ciudad de Medellín, una de las zonas de mayor exposición a desastres en el municipio. En este artículo se presentan la metodología, los materiales usados y los resultados obtenidos.

\section{Metodología}

Se planteó una metodología que permite el cálculo del riesgo definido mediante la ecuación:

$$
R=P[T] \times P[C \mid T] \times u(C)
$$

Donde $R$ es el riesgo, $P[T]$ es la amenaza entendida como la probabilidad de que ocurra un evento amenazante, $P[C \mid T]$ es la vulnerabilidad entendida como la probabilidad condicional de que se produzcan daños considerando que ya ocurrió una falla y $u(C)$ es el costo de las consecuencias. Para el caso de los deslizamientos, la $P[T]$ se entenderá como la probabilidad de falla, es decir la probabilidad de que el talud falle, que en este caso corresponde a la probabilidad de falla total (Pft). Aunque la falla se podría dar solo por efecto de la gravedad, lo más común es que sea desencadenada por un agente detonante como los sismos o la lluvia.

Para la evaluación de la amenaza se elaboró un modelo de cálculo, que se muestra gráficamente en la Figura 1, y que permite calcular la probabilidad de falla mediante un índice de confiabilidad (Ecuaciones 2 y 3). Se calcula de dos formas, la primera como la probabilidad de que los valores considerados límites sean excedidos. En el caso del factor de seguridad por deslizamiento (FSD), la probabilidad de falla se determina como la probabilidad de que FSD sea menor que la unidad (Ecuación 2) y la segunda como la probabilidad de que la aceleración generada por un sismo $(A h)$ supere un parámetro denominado aceleración crítica $(A c)$, es decir $F S D=P\left(A_{h}>A_{c}\right)$. 


$$
\begin{gathered}
\beta=\frac{E\lfloor F S D]-1}{\sigma[F S D]} \\
\theta=\frac{\left(A_{C}-A_{h}\right)}{\sigma A_{C}}
\end{gathered}
$$

Donde $E[F S D]$, es el valor determinista del FSD calculado con los valores medios de las variantes individuales y $\sigma[F S D]$ es la variación estándar de $F S D$, considerando que el valor crítico de FSD es 1.0 (Christian et al., 1994; Baecher y Christian, 2003). $A c$ es la aceleración crítica, $A h$ es la aceleración del sismo de diseño o del sismo más probable en la zona de estudio y $\sigma A c$ es la desviación estándar de la aceleración crítica. Los índices $\beta$ y $\theta$ están relacionados con la probabilidad de falla, lo que posibilita una evaluación más firme de la estabilidad. Es de anotar que las Ecuaciones 2 y 3 sólo son válidas si la Función de Distribución de Probabilidad (FDP) del FSD es normal. Cuando la distribución de probabilidad es diferente a la normal, se deben usar otras formulaciones (Christian et al., 1994, Baecher y Christian, 2003).

La aceleración crítica se calcula teniendo en cuenta el efecto de los sismos por medio del Método de Newmark (1965), el cual se basa en un modelo de estabilidad de talud infinito. Según este método, para evaluar la estabilidad por deslizamientos detonados por sismos, se calcula la aceleración necesaria para producir dicho movimiento, denominada aceleración crítica $(A c)$. Para esto se recurre a métodos seudoestáticos en los cuales la fuerza debida al sismo se añade al modelo como una fracción del peso de la masa que se desliza. A continuación se presentan las expresiones resultantes para el modelo de talud infinito que será usado en este trabajo:

$$
A_{c}=(F S D-1) \operatorname{sen} \alpha
$$

Donde $\alpha$ es el ángulo de inclinación promedio del terreno $\left[^{\circ}\right]$ y $F S D$ se determina con la expresión:

$$
F S D=\frac{c}{\gamma H\left(\sin \alpha+A_{h} \cos \alpha\right)}+\frac{\left(\gamma H-\gamma_{w} H_{w}\right) \cos \alpha \tan \varphi}{\gamma H\left(\sin \alpha+A_{h} \cos \alpha\right)}
$$

Donde $A h$ es la aceleración producida por el sismo dada como múltiplo de la aceleración de la gravedad $g, H$ es el espesor de la zona que falla [m], $H w$ es la altura del agua medida desde la superficie de falla $[\mathrm{m}]$, c es la cohesión del suelo $[k P a], \varphi$ es el ángulo de fricción interna del suelo [ $\left.{ }^{\circ}\right], \gamma$ es el peso unitario del suelo $\left[\mathrm{kN} / \mathrm{m}^{3}\right], \gamma w$ es el peso unitario del agua $\left[\mathrm{kN} / \mathrm{m}^{3}\right]$. 
En la región del VA, los deslizamientos se dan con mayor frecuencia en temporadas lluviosas, en las cuales se presenta un aumento de la saturación del suelo con la consecuente disminución de su cohesión y el incremento de la presión de poros. El proceso de disminución en la resistencia al cortante debido a los cambios de contenido de agua es un proceso altamente complejo que no fue considerado en el desarrollo de este estudio. Por lo tanto, el efecto de la saturación se tuvo en cuenta solamente como el aumento de la presión del agua, y para efectos de análisis en este trabajo se consideraron dos situaciones relacionadas con el nivel de agua desde la superficie de falla $(H w)$, una en la cual el nivel del agua está en la condición más crítica, es decir $H w=H$, y otra favorable en la cual $H w=0$.

La eventual saturación del suelo es un fenómeno aleatorio a tener en cuenta en la evaluación de la probabilidad de movimientos en masa. En este caso se consideró la probabilidad de que el suelo se encuentre saturado o no. De acuerdo con el teorema de probabilidad total, la probabilidad de falla total de un talud está dada por la siguiente ecuación:

$$
P_{f t}=P_{f s} \times P_{s}+P_{f n s} \times\left(1-P_{s}\right)
$$

Donde $P f t$ hace referencia a la probabilidad de falla total, $P f s$ es la probabilidad de falla del talud debido a la acción del sismo en condición saturada, Pfns es la probabilidad de falla en condición no saturada, $P S$ es la probabilidad marginal de que el suelo esté saturado y $(1-P S)$ equivale a la probabilidad marginal de que el suelo no esté saturado.

La probabilidad de falla de los taludes en condición saturada y no saturada se puede calcular independientemente, pero determinar la probabilidad de que el suelo se encuentre saturado es difícil, debido a lo complejo del fenómeno de variación de las condiciones de contenido de agua del suelo. Para el caso de los suelos del VA la información es limitada para determinar la probabilidad de que los suelos estén saturados, sin embargo, en trabajos realizados por diferentes autores (Moreno et al., 2006; Hidalgo y Vega, 2012) se ha podido establecer que la mayoría de los deslizamientos se generan por la saturación de los suelos debido al efecto de la lluvia acumulada, y que la ocurrencia de movimientos en masa es posible relacionarla con la cantidad de lluvia mediante los llamados umbrales de falla.

En este trabajo se considera que la probabilidad de saturación del suelo está enlazada con la posibilidad de que el umbral de falla sea excedido. Esta consideración parte de aceptar que la condición dada por el umbral de falla representa una situación de saturación favorable para los deslizamientos, con la ya mencionada reducción de la resistencia al cortante de los materiales debido a la disminución de la succión y la generación de presiones de poros (Hidalgo y Veja, 2012; Veja, 2013; Hidalgo, 2013). Posteriormente al determinar la probabilidad de que el suelo se 


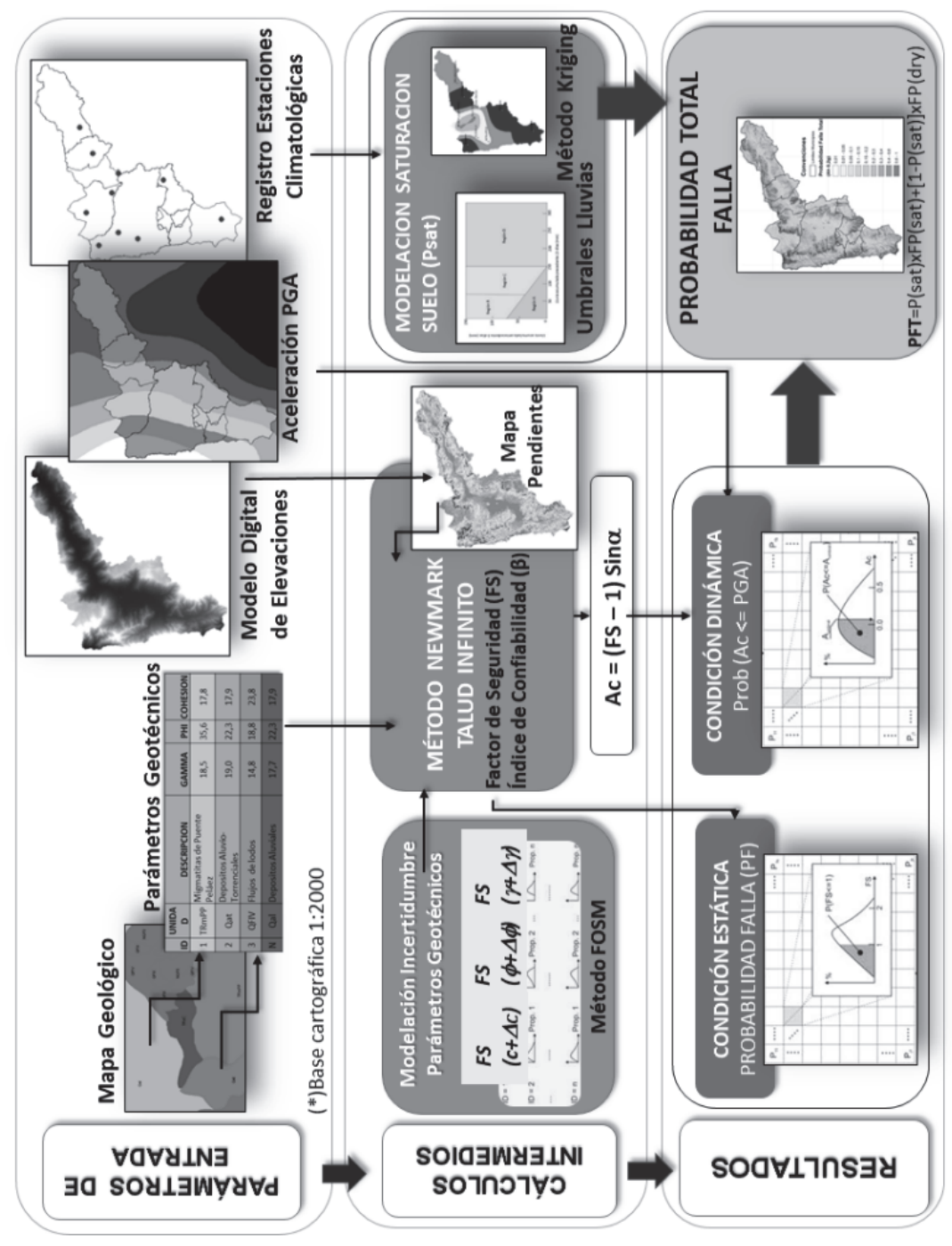


118 Johnny Alexander Vega Gutiérrez $\quad$ Estimación del riesgo en edificaciones por deslizamientos...

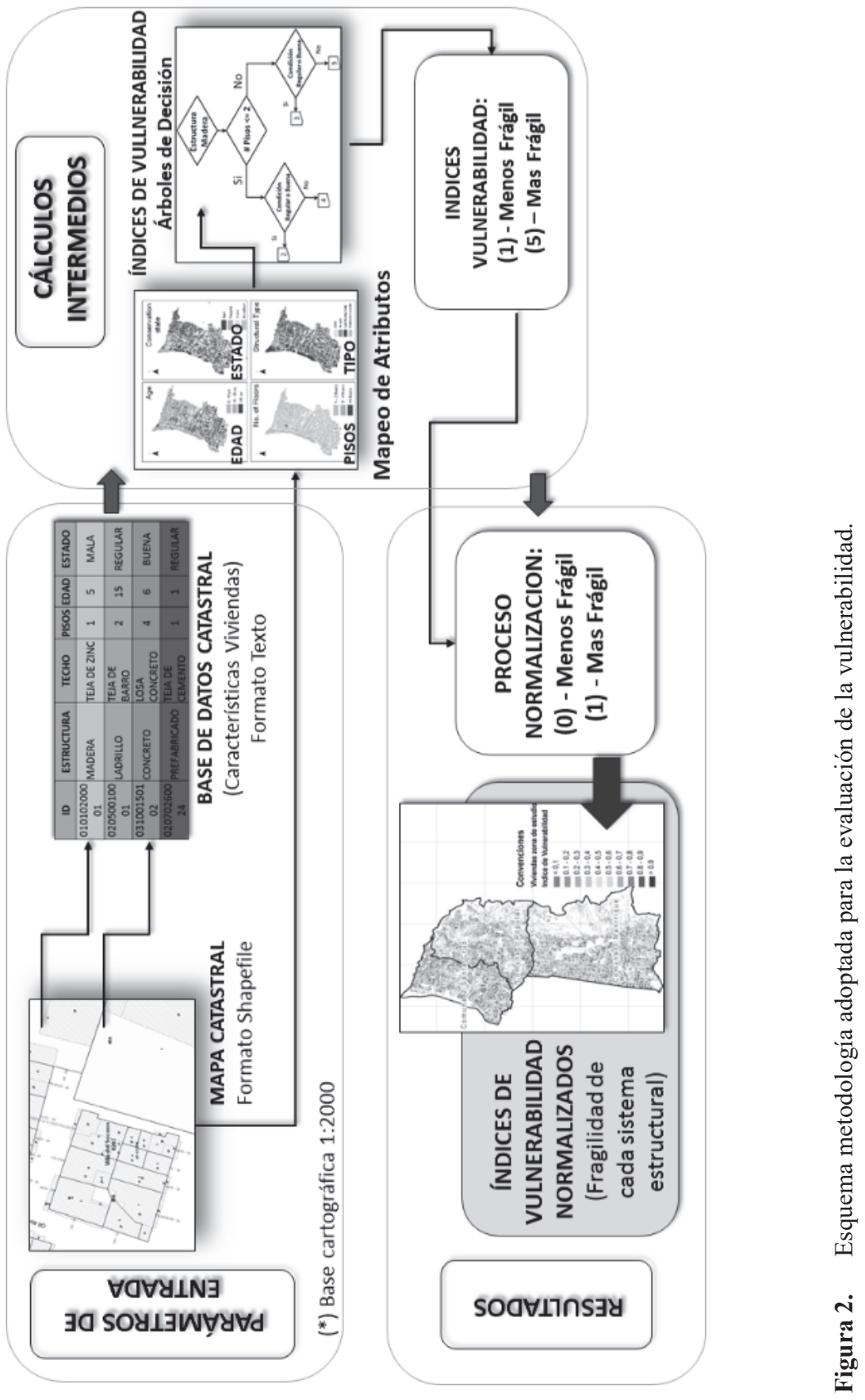


encontrará saturado según los datos de (10) estaciones pluviográficas, se desarrolló un proceso geoestadístico para estimar por interpolación la probabilidad de saturación en cada una de las celdas. El método de interpolación usado en este trabajo corresponde al Método de Kriging.

La vulnerabilidad se evaluó a partir de la metodología resumida en la Figura 2, y considera la definición del nivel de daño de las edificaciones a partir un índice calculado mediante el uso de árboles de decisión, uno por cada tipo de material posible para la estructura principal de las edificaciones, los cuales permiten asignar a cada variable un valor y a todas las combinaciones, para llegar a un valor final que indica cuan frágil o susceptible a daño es cada edificación. Este índice se basa en la calificación de (5) factores o atributos que tienen relación con la fragilidad del sistema estructural de las edificaciones para atender las solicitaciones de un evento sísmico y de un posible deslizamiento, el cual refleja la interacción entre el tipo de estructura y algunas de sus características.

Los cinco atributos mencionados, se obtuvieron de la base de datos de Catastro Municipal, los cuales fueron procesados para obtener un registro por cada vivienda, en función del uso y tipo de vivienda y material más predominante. Los atributos considerados corresponden a: el tipo de sistema estructural, el estado de conservación, el número de niveles de pisos, el tipo de cubierta (techo), y la edad o vetustez de la edificación. Una vez procesada y depurada la base de datos, se obtuvo un único índice que agrupa las condiciones menos favorables de cada atributo, desde el punto de vista de la vulnerabilidad para cada parcela, bajo un escenario en el cual las edificaciones estén directamente asentadas sobre el bloque o masa de suelo deslizante.

El indicador de vulnerabilidad física de las edificaciones de la zona de estudio, se calculó mediante el uso de árboles de decisión (Figura 3), en los cuales el criterio adoptado indica que el valor más bajo (1) corresponde a una mejor condición y el valor más alto (5) corresponde a una peor condición bajo el escenario más desfavorable. Para el caso de los sistemas estructurales de ladrillo y concreto con una cubierta de losa de entrepiso en concreto reforzado, se consideró una reducción de (0.5) en el valor del indicador de vulnerabilidad, puesto que el efecto de diafragma generado por la losa de entrepiso, contribuye a la rigidez de la estructura, reduciendo en cierta medida su fragilidad o susceptibilidad al daño. Esto se consideró para los sistemas estructurales mencionados, dado que son quienes tienen la capacidad de soportar el peso de una cubierta de tal tipo. Hecho esto, el índice fue normalizado (Figura 4) para obtener valores en un rango de cero (0) a uno (1), de manera que pudiera ser compatible con el rango adoptado para la amenaza, en este caso el deslizamiento de masas de tierra, la cual se valora de cero a uno, puesto que corresponde a una probabilidad. 


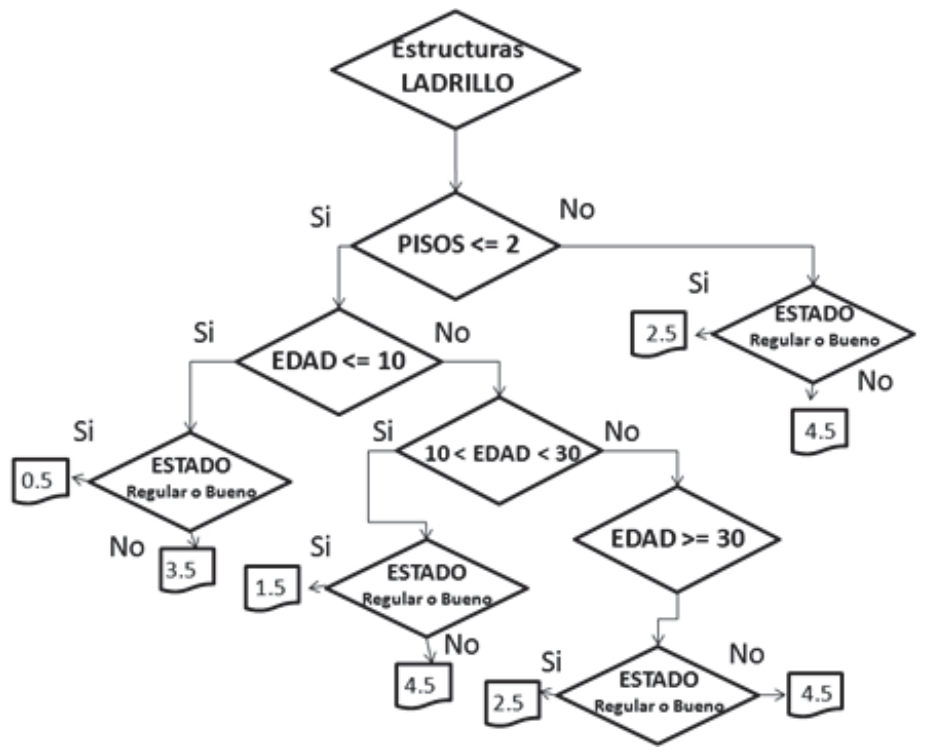

Figura 3. Árbol de decisión para la estimación del índice de vulnerabilidad para viviendas con estructura de ladrillo (con cubierta en losa de concreto).

El riesgo corresponde al número de pérdidas humanas, heridos, daños a las propiedades y efectos sobre la actividad económica, debido a la ocurrencia de un evento desastroso que se denomina amenaza, y a su efecto sobre los elementos expuestos según su grado de vulnerabilidad.

De acuerdo a lo planteado mediante la Ecuación 1, es posible obtener el riesgo asociado a los daños que puede llegar a ocasionar un deslizamiento de masa accionado por un evento sísmico en las viviendas de la zona de estudio. Para ello, fue preciso determinar los índices de daño, calculados como el producto de la probabilidad de falla total por el índice de vulnerabilidad. Una vez que se han calculado los índices de daño y se consideran los costos suscitados por un eventual desastre a partir de los valores de las viviendas obtenidos de los registros catastrales, se obtuvo el riesgo de las edificaciones por la probabilidad de falla en la zona de estudio.

Para implementar la metodología propuesta, se elaboró un modelo cálculo de estabilidad de laderas para la determinación de la amenaza de forma probabilísta, usando para esto el lenguaje de programación Python y su respectiva implementación en el software ArcGISTM.

Para cada uno de los parámetros involucrados en el análisis de estabilidad de las laderas, se programó su respectiva ecuación de cálculo como un submodelo, es decir, se implementaron submodelos para el factor de seguridad, aceleración crítica, probabilidad de falla, índices de vulnerabilidad, índices de daño y riesgo, entre 
otros submodelos auxiliares. Así mismo, cabe resaltar que se emplearon diferentes herramientas de análisis espacial y geoprocesamiento, de manera que a partir de la información base (descrita en la sección 3) se obtuviera información para incorporar en los submodelos mencionados, permitiendo explorar y analizar diferentes escenarios. En la Figura 5, se presenta el esquema típico de un submodelo implementado que corresponde en este caso a la aceleración crítica $(A c)$.

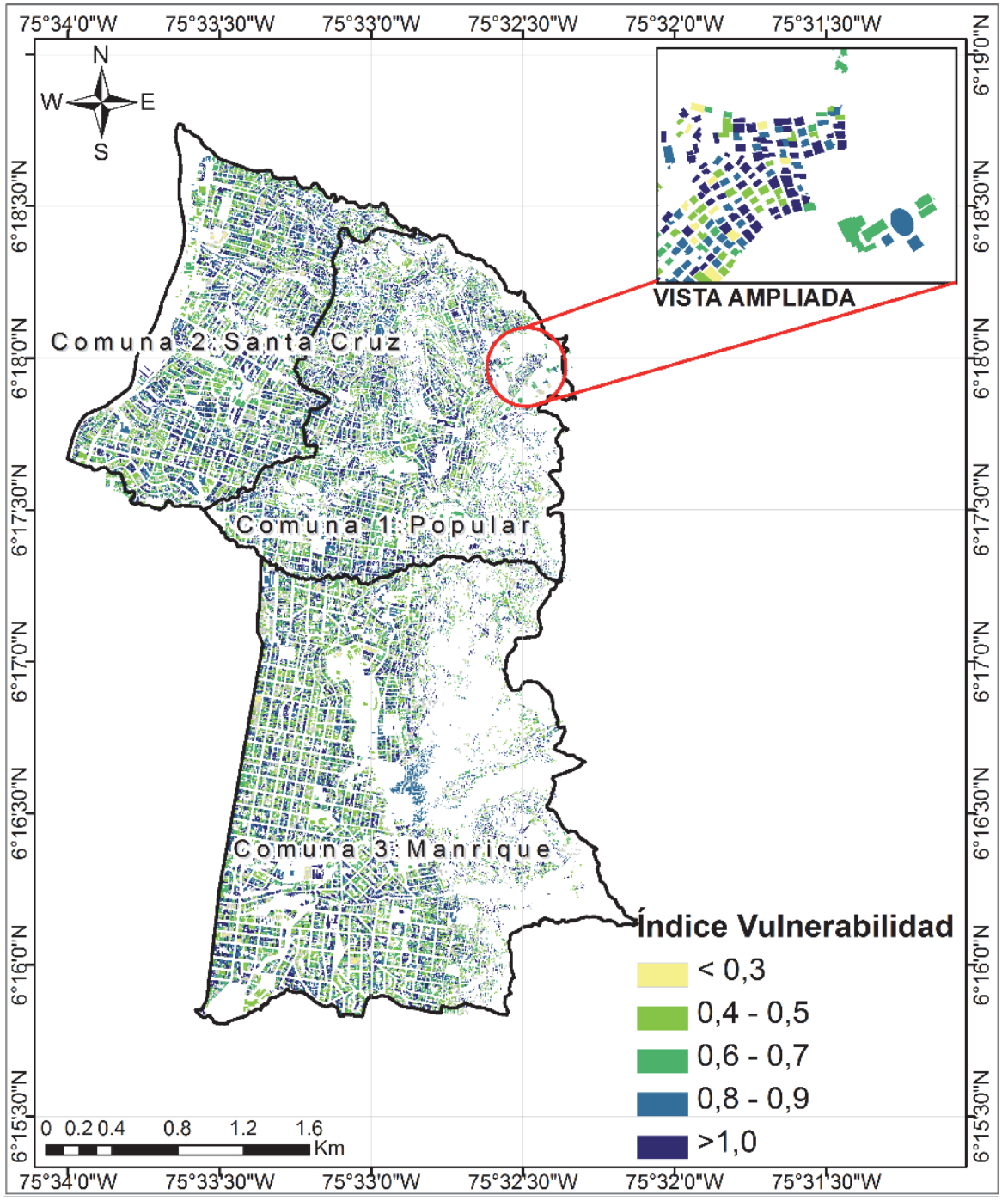

Figura 4. Índice de Vulnerabilidad Normalizado de las viviendas de la zona de estudio. 


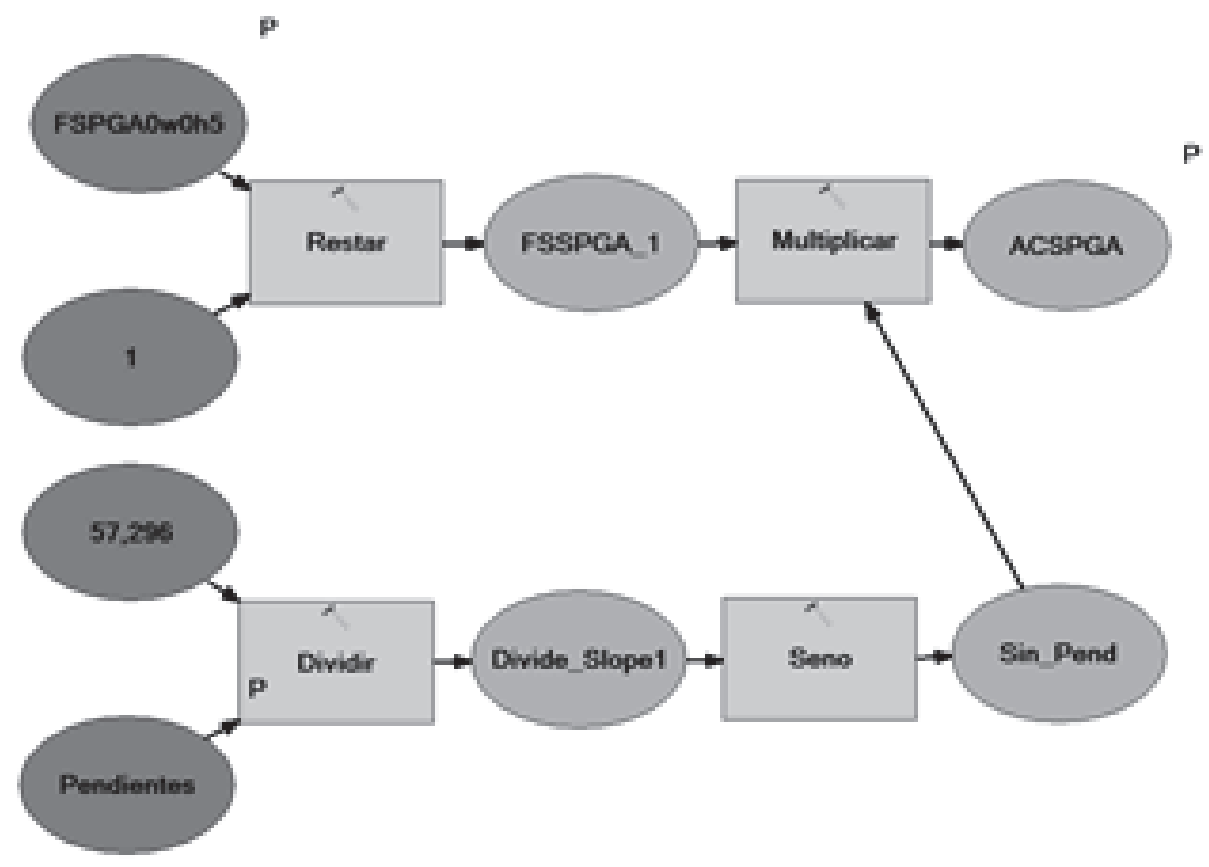

Figura 5. Submodelo implementado para el cálculo de la aceleración crítica.

\section{Caso de estudio}

La metodología propuesta se usó para estimar el riesgo por deslizamiento de una zona de la ciudad de Medellín que tradicionalmente ha presentado emergencias por movimientos en masa. En la Figura 6, se muestra la delimitación de la zona de estudio para el desarrollo de esta investigación, cuya localización se encuentra centrada en las coordenadas $6.25^{\circ} \mathrm{N}, 75.6^{\circ} \mathrm{W}$.

La información requerida por el modelo implementado bajo ambiente SIG, corresponde a una cuadricula regular de celdas para cada variable temática. Para este caso se usaron celdas cuadradas de $50 \mathrm{~m}$ de lado. La información usada en el modelo se describe a continuación:

- Modelo Digital de Elevaciones (DEM): el cual fue obtenido originalmente de AMVA (2007), y cuenta con una mejor resolución espacial. Mediante un proceso de remuestreo se adaptó a la escala de trabajo de las demás capas. Para este trabajo, este proceso resulta adecuado dado que la aplicación de un DEM de mejor resolución para zonas con falta de datos o alta incertidumbre sobre las propiedades físicas del suelo es ineficiente debido a su baja relación costobeneficio. 


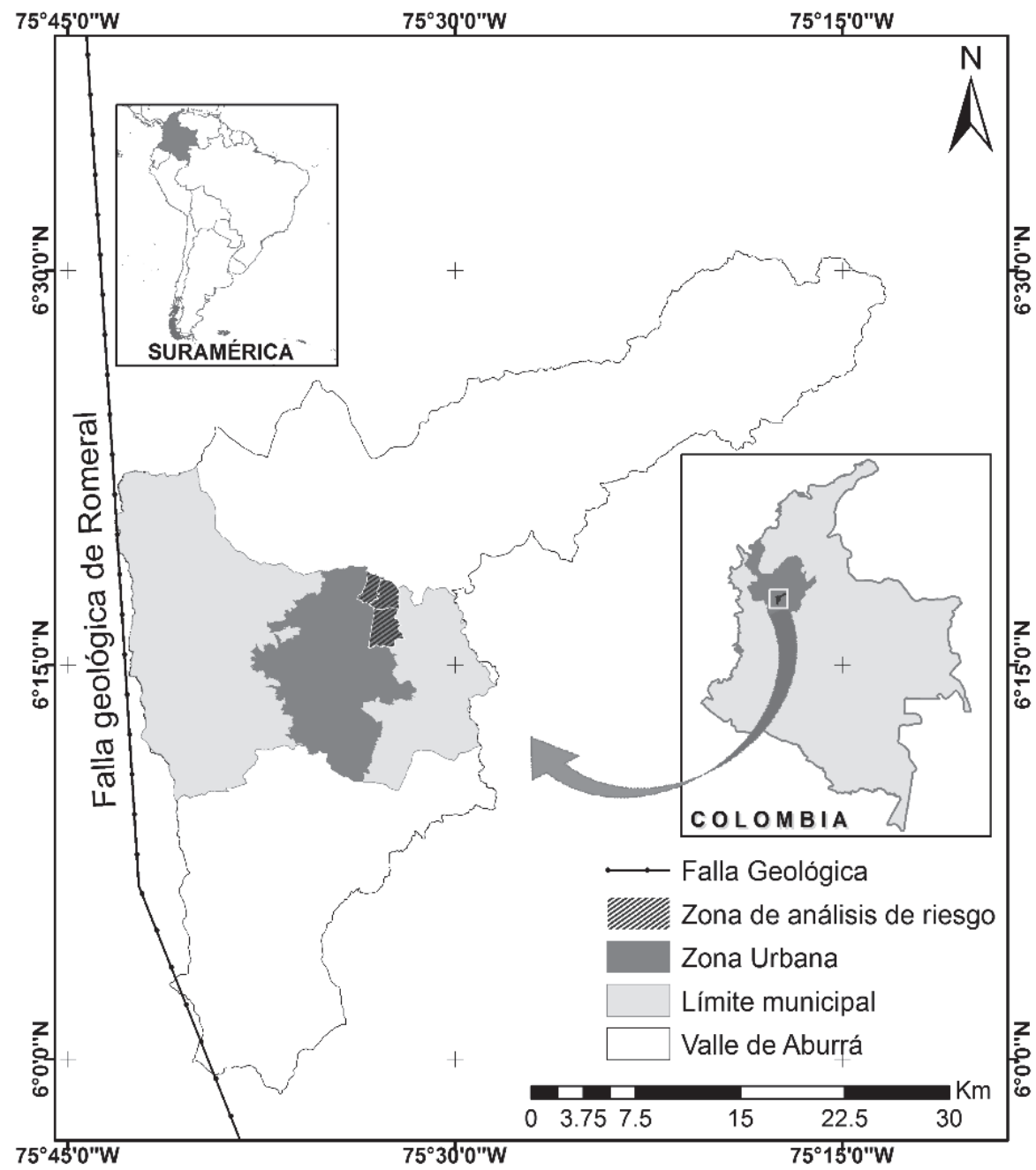

Figura 6. Localización de la zona de estudio.

- Datos geológicos y propiedades del suelo: el VA y sus alrededores, en relación con la composición de las rocas, presenta gran cantidad de rocas metamórficas como esquistos, anfibolitas, migmatitas y gneises; rocas ígneas como granodioritas, dunitas, gabros y basaltos; rocas volcano-sedimentarias y los depósitos de origen aluvial y de vertiente, además de los llenos de origen antrópico (AMVA 2007). Según la información presentada en Hidalgo (2013) e Hidalgo y Vega (2012), a cada uno de estos suelos se le atribuyeron los parámetros de resistencia y peso unitario. 
- Datos de sismicidad: el VA se encuentra en una zona de amenaza sísmica entre media y alta (AIS, 2010) y las principales sismo fuentes se presentan en el sistema de falla Cauca-Romeral y otros sistemas menores (AMVA, 2007). En este estudio, se trabajó un escenario dado por la distribución de aceleraciones determinada por la microzonificación sísmica del VA (AMVA, 2007), y otro con una aceleración uniforme de $0.2 \mathrm{~g}$, establecido por la Norma Colombiana de Construcciones Sismo Resistentes NSR-10 (AIS, 2010) para la ciudad.

- Datos de precipitación: hidrológicamente, el VA se caracteriza por un régimen de lluvias que tradicionalmente ha sido bimodal, con dos épocas lluviosas aproximadamente en los meses de marzo-abril-mayo y septiembre-octubrenoviembre. Los mayores valores de precipitación están entre 2,800 y 3,200 $\mathrm{mm} / \mathrm{año}$, y se presentan en la parte norte y sur de la cuenca. Las menores precipitaciones, con valores entre los 1,400 mm/año y 1,800 mm/año, se dan en la zona central de la cuenca y se extienden hacia la zona occidental (AMVA, 2009). Se utilizaron los datos de precipitación acumulada diaria de 10 estaciones pluviométricas localizadas en el VA con series de registros entre 13 y 50 años.

- Información catastral de las viviendas: el punto de partida para la estimación del índice de vulnerabilidad de las viviendas consistió en la adquisición de la base de datos cartográfica y alfanumérica de los predios correspondientes al sector nororiental de la ciudad de Medellín. Para ello, se hizo uso de la información suministrada por la Subsecretaría de Catastro Municipal.

\section{Resultados}

La aceleración crítica en cada celda se determinó considerando la superficie de falla a $5 \mathrm{~m}$ de profundidad y condiciones de agua coincidentes con la superfície de falla $(\mathrm{Hw}=0)$ y del terreno $(\mathrm{Hw}=5 \mathrm{~m})$ respectivamente. La aceleración crítica en condiciones húmedas varía entre 0 y $1.13 \mathrm{~g}$ y en condiciones saturadas varía entre $0 \mathrm{y}$ $0.84 \mathrm{~g}$. Igualmente se usando la Ecuación 3 se calculó el coeficiente $\theta$ para los valores de Ah establecidos en la microzonificación sísmica de la ciudad (AMVA, 2007), bajo un escenario de condición húmeda $(\mathrm{Hw}=0)$ y saturada $(\mathrm{Hw}=5 \mathrm{~m})$.

Suponiendo una distribución normal, se calculó la $P f t$ de cada celda (Figura 7a) encontrando una variación entre 0.15 y 1.0 presentándose las mayores probabilidades de falla en áreas caracterizadas por pendientes superiores al $40 \%\left(22^{\circ}\right)$ y condiciones hidrológicas desfavorables como las que se presentan en el noroccidente en el corregimiento de Palmitas y en la zona sur oriental. Las áreas localizadas en la zona central del VA que tienen pendientes más suaves, menores al 40\% presentan probabilidades de falla entre 0.4 y 0.5 y representan aproximadamente el $86 \%$ del área estudiada. 

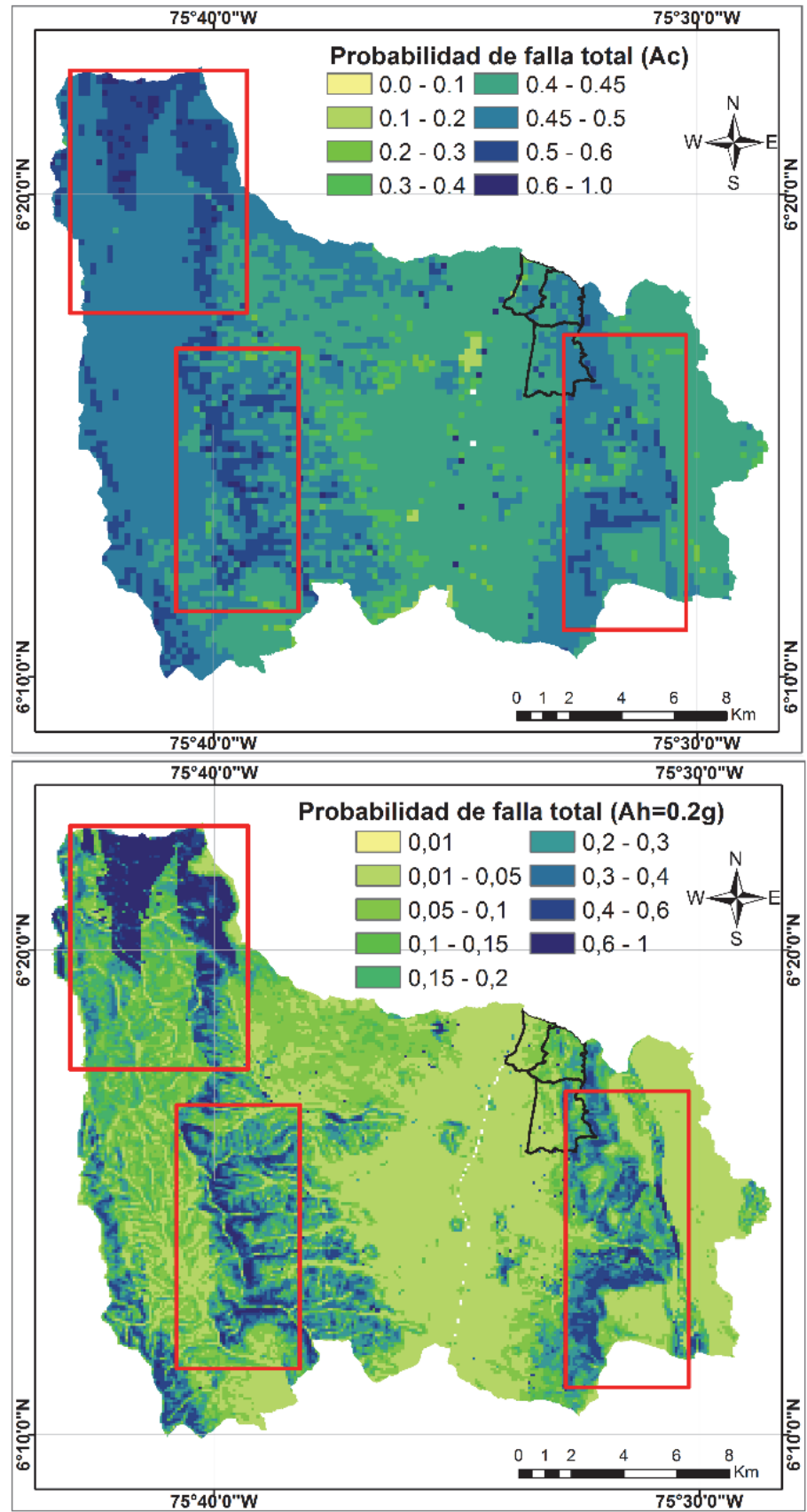

Figura 7. (a) Distribución de la probabilidad de falla total $\mathrm{P}(\mathrm{Ah}>\mathrm{Ac})$. (b) Distribución de la probabilidad de falla total para $\mathrm{P}(\mathrm{FSD}<1.0)$ con $\mathrm{Ah}=0.2 \mathrm{~g}$ 
Estas probabilidades de falla están dadas para un sismo con una posibilidad de excedencia de $10 \%$ en 50 años, lo cual denota que las probabilidades anuales de que se produzca un deslizamiento por un sismo cambian entre $3 \times 10-4$ y $2 \times 10-3$. De acuerdo con la distribución que presentan las probabilidades de falla y considerando que la amenaza por deslizamiento puede ser clasificada como muy alta (Pf anual $>0.2$ ), Alta (0.02-0.2), media (0.002-0.02), bajo (0.002-0.0002), muy bajos $(<0.0002)$, el VA tiene el $99 \%$ de su territorio en condiciones de amenaza por deslizamientos originados por sismos baja a muy baja y un $1 \%$ en condiciones medias de amenaza.

Usando la Ecuación 2, se calculó el coeficiente $\beta$ para valores de $A h$ de $0.2 \mathrm{~g}$, para un escenario en condición húmeda $(\mathrm{Hw}=0)$ y en condición saturada $(H w=5 \mathrm{~m})$. Considerando una distribución normal, se calculó la Pft de cada celda (Figura 7b) encontrando una variación entre 0.1 y 1.0 , presentándose una distribución similar a la observada para la $A c$, cuyas condiciones más desfavorables se dan en el noroccidente, corregimiento de Palmitas, y en la zona sur oriental del VA, y las condiciones más estables, en las áreas localizadas en la zona central. Los rangos de valores de las probabilidades de falla y su distribución son comparables constituyendo zonas de amenaza similares a las establecidas anteriormente en las que predominan las zonas de baja y muy baja amenaza.

En el escenario estimado con una aceleración sísmica de $0.2 \mathrm{~g}$, las máximas probabilidades de que ocurra un deslizamiento, corresponden a un $99.96 \%$ en una condición de húmeda normal del suelo y al $100 \%$, en una condición de saturación total del suelo. Las áreas que presentan estos valores de probabilidad de falla, corresponden a zonas de los corregimientos de Palmitas, San Cristóbal, Altavista y Santa Elena, como se indica en los recuadros de la Figura 7.

Igualmente para corroborar la capacidad del modelo para determinar zonas críticas, se identificaron los sitios que presentan las peores condiciones de estabilidad, determinados como aquellas celdas que presentaron FSD menores que 1.2 en condiciones saturadas y con aceleración de $0.2 \mathrm{~g}$ o con las mayores probabilidades de falla total. Se observa que los puntos críticos identificados están localizados en zonas que frecuentemente son afectados por problemas como los descritos en trabajos como el proyecto "Amenaza, vulnerabilidad y riesgo por movimientos en masa, avenidas torrenciales e inundaciones en el Valle de Aburrá”, y que se muestran en la Figura 8.

Para efectos de verificar la incidencia y correlación de la geomorfología y la geología estructural de la zona con los puntos críticos mencionados anteriormente, se identificaron algunas fracturas y discontinuidades superficiales a partir de la fotointerpretación y procesamiento de una escena del año 2013 del satélite Landsat 8 y del análisis del DEM, con las cuales se pudo comprobar que existe una fuerte 


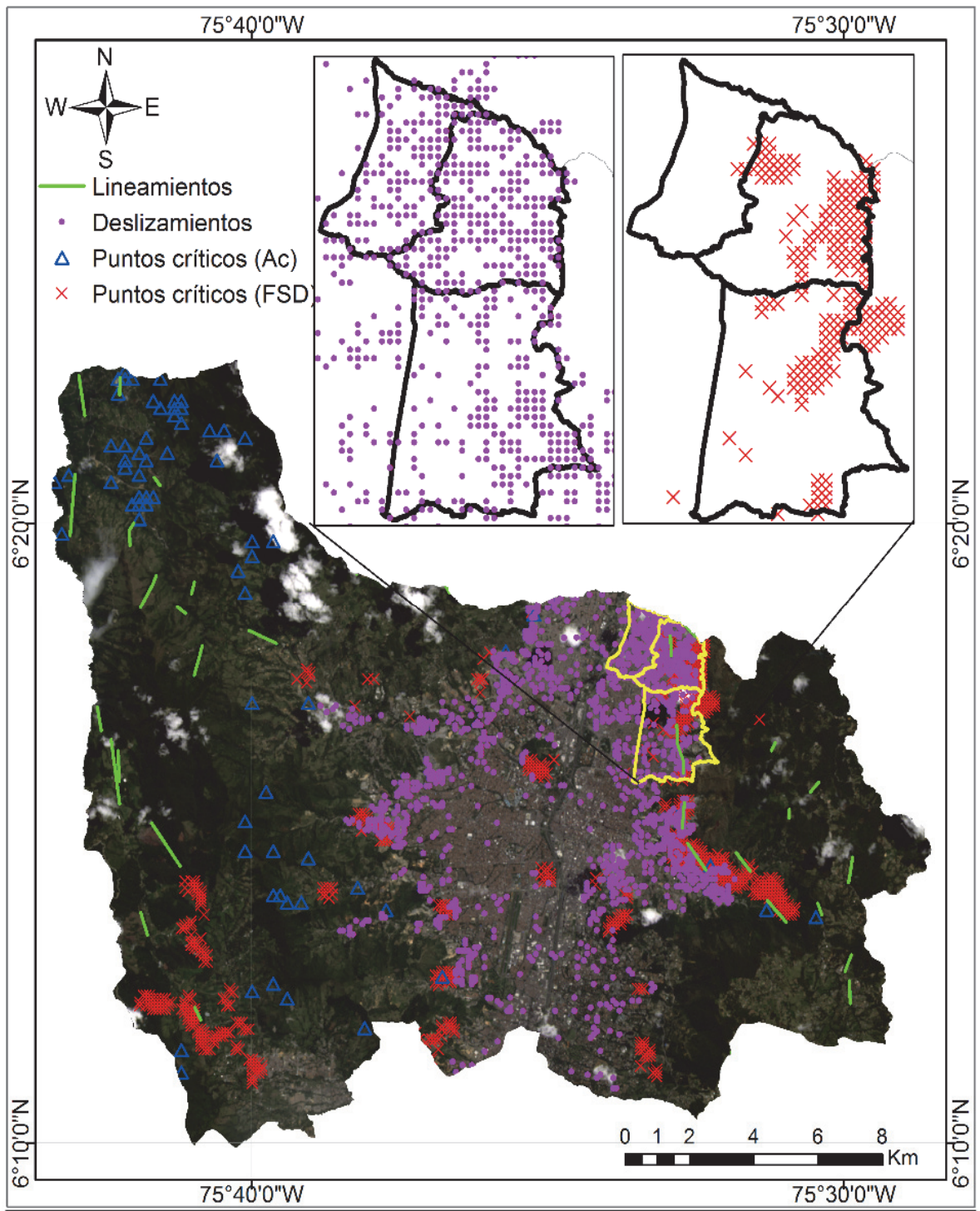

Figura 8. Localización de puntos críticos calculados con el modelo y de los procesos morfodinámicos ocurridos en el Municipio de Medellín (1985-2006).

correlación espacial de los lineamientos estructurales identificados con respecto a los puntos críticos calculados, lo cual puede apreciarse en la Figura 8, permitiendo establecer que las geoformas y el escarpe erosivo característico de la ladera oriental del VA influye en la estabilidad de dichas laderas. 
En la Figura 8, se pueden ver las zonas obtenidas mediante un análisis espacial donde los puntos críticos calculados muestran un porcentaje de coincidencia respecto al inventario antes mencionado, del orden de $61 \%$ para el caso de los puntos críticos por FSD. Para el caso de los puntos críticos por $A c$ se obtuvo un $13 \%$ de coincidencia, lo cual representa un escenario de un sismo con una aceleración de $0.2 \mathrm{~g}$ y que serían puntos donde el factor seguridad estaría por debajo de la unidad.

En cuanto a la vulnerabilidad, en la Figura 4 se presentaron los resultados obtenidos para el Índice de Vulnerabilidad, el cual considera el efecto de diafragma que generan las cubiertas de entrepiso de concreto, en el comportamiento estructural de las viviendas, tal cual se indicó anteriormente. Se observa que debido a la influencia conjunta de condiciones desfavorables de cada atributo considerado, desde el punto de vista de la fragilidad estructural de las viviendas de la zona de estudio se presentan altos valores de vulnerabilidad en todo el sector nororiental de la ciudad.

En la Figura 9b se puede apreciar que los valores más altos del índice de riesgo dentro de la zona de estudio se presentan en el costado oriental, que corresponden efectivamente a las zonas de mayores pendientes con valores de vulnerabilidad altos. Así mismo, en el mismo costado se observa correspondencia con los valores obtenidos de índice de daño mostrados en la Figura 9a.

A parte de calcular los parámetros de interés con un valor de $\mathrm{Ah}$ de $0.2 \mathrm{~g}$, se realizaron varias simulaciones para otros valores de $\mathrm{Ah}(0,0.05,0.1,0.3,0.5,0.7 \mathrm{y}$ 1.0), con el objetivo de modelar el efecto del sismo deflagrador, analizar la influencia y variabilidad presentada en los costos suscitados por un eventual deslizamiento accionado por dicho sismo. En la Figura 10, se puede apreciar el resultado de las simulaciones mencionadas anteriormente, donde se presenta la curva de los costos suscitados por la probabilidad de eventos catastróficos en la zona de estudio, la cual considera la pérdida máxima probable, bajo el escenario estructural real de las edificaciones. En dicha gráfica se puede observar la pérdida esperada en millones de dólares, para cada valor de aceleración horizontal del suelo considerado en el desarrollo del presente trabajo.

\section{Conclusiones}

El modelo elaborado posibilita estimar la amenaza y la zona de influencia debido a movimientos en masa detonados por sismos, estimando la influencia de las condiciones de saturación debidas a las lluvias. Los resultados encontrados muestran que el modelo es robusto en la identificación de zonas críticas para la estabilidad. Con esto se puede hacer una priorización de zonas que deben ser estudiadas en detalle para garantizar la seguridad de las personas e infraestructuras próximas al sitio.

Cabe resaltar la pertinencia de una herramienta computacional como los SIG en este tipo de investigaciones aplicadas, puesto que permiten implementar y modelar 


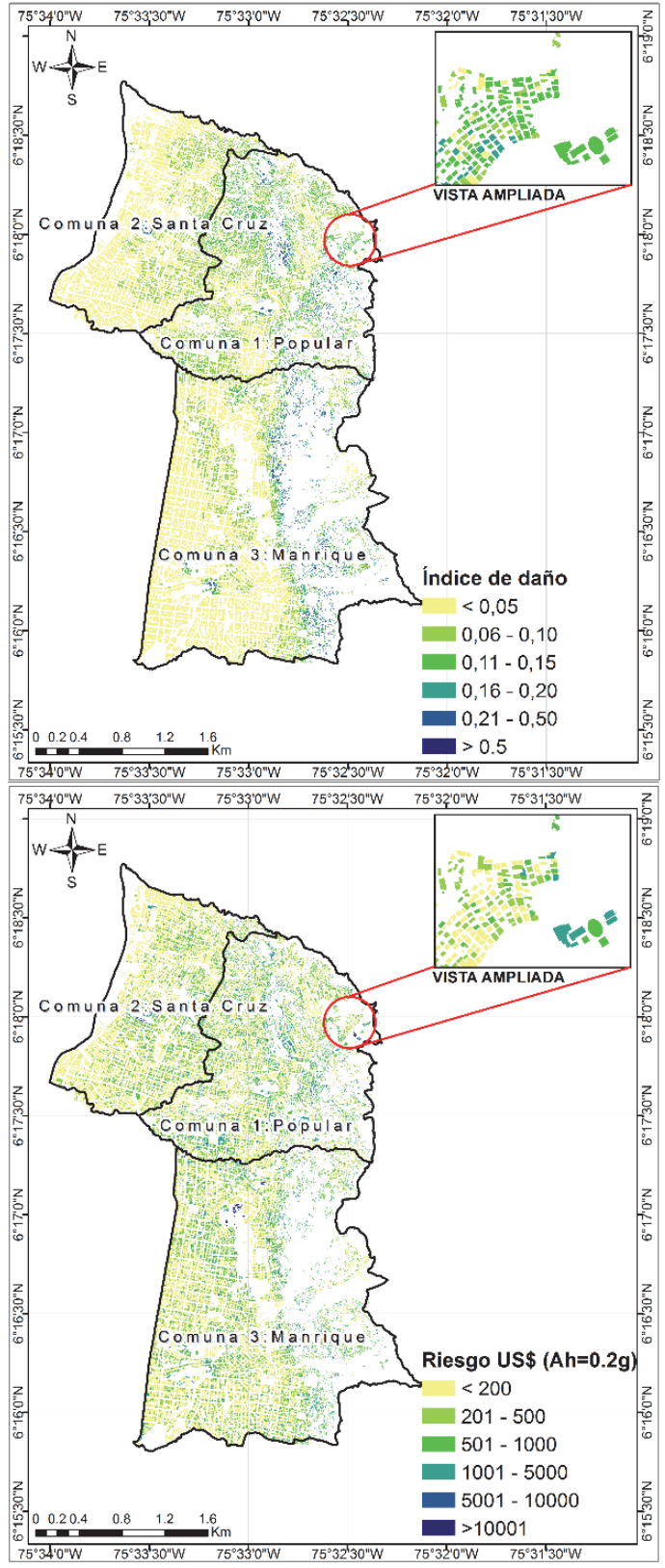

Figura 9. (a). Índice de daño de las edificaciones en la zona de estudio. (b) Riesgo suscitado por la probabilidad de falla de las edificaciones en la zona de estudio dado que se presenta un deslizamiento como consecuencia de un evento sísmico. 


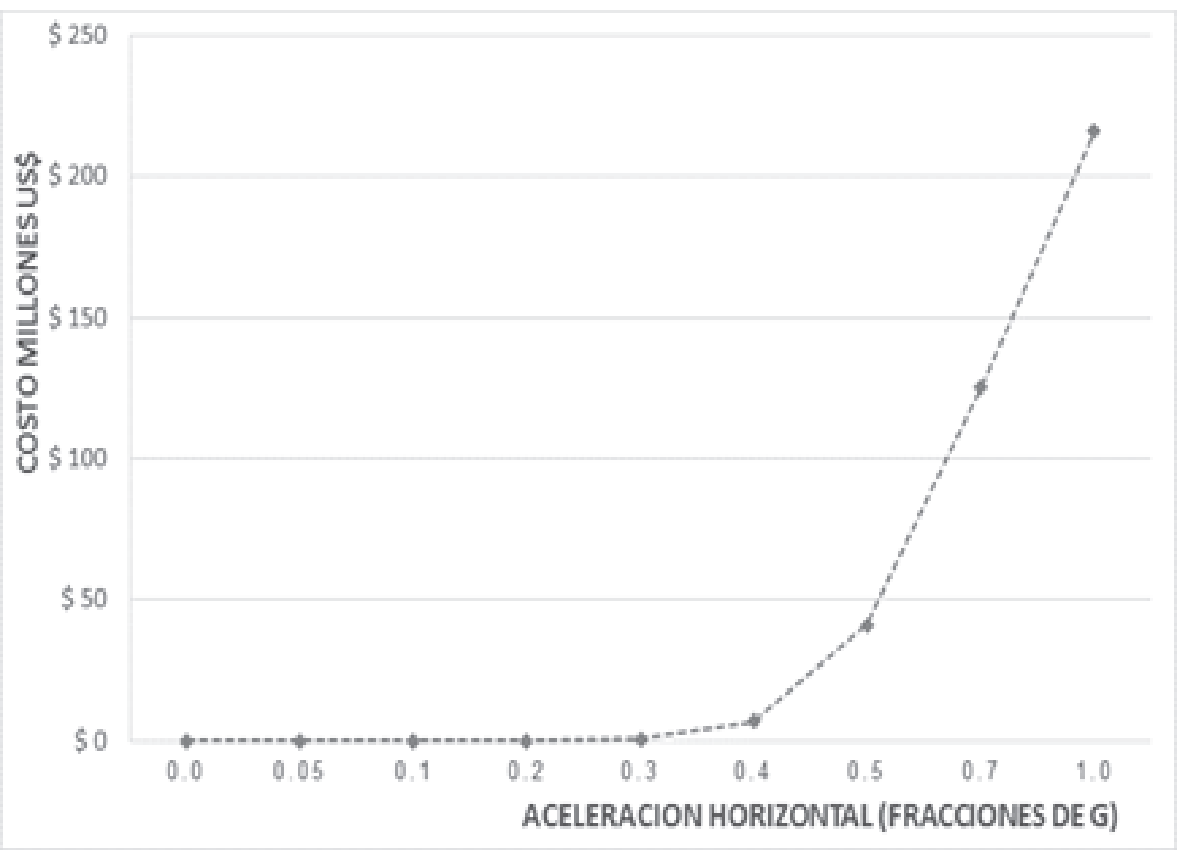

Figura 10. Costos suscitados por la probabilidad de eventos catastróficos. Pérdida máxima probable según el índice de daño (ID).

varios escenarios (por ejemplo simular diferentes valores de PGA, considerar la variabilidad espacial de los parámetros geomecánicos, considerar diferentes marcos de vulnerabilidad, entre otros), que involucran una gran cantidad de variables, permitiendo modificar fácilmente los parámetros involucrados y ejecutar rápidamente los procesos de análisis espacial, lo cual significa un ahorro significativo de tiempo y capacidad de almacenamiento de la información.

La adopción de sistemas computarizados de este tipo, permiten su inclusión como precursor o potencializador de sistemas de alerta temprana para la evaluación, atención y prevención de desastres, principalmente en entidades municipales, con lo cual se busca reducir al mínimo la pérdida de vidas humanas principalmente, así como la minimización de daños en la infraestructura urbana.

Con el modelo implementado se logró cuantificar el riesgo ocasionado por este tipo de desastre en una zona de la ciudad considerando diferentes valores de $A h, \mathrm{y}$ además, se realizó un análisis de los costos suscitados por los daños en las viviendas del sector de estudio. Así, es posible determinar el monto de la inversión a realizar para disminuir el riesgo de las viviendas expuestas a un evento desastroso a un nivel admisible de seguridad, dado que para un mismo valor de $A h$, por ejemplo la máxima esperada para una ciudad en un periodo de retorno determinado, se puede 
determinar la diferencia en costo entre el escenario estructural real de las viviendas expuestas, respecto a un escenario supuesto que cumpla con la normativa en construcción sismo resistente de cada país.

En síntesis, es clara la utilidad de las herramientas geomáticas para la temática de la gestión del riesgo, pues en cada fase del análisis, desde la captura de la información, su procesamiento, análisis y modelaciones, hasta la presentación de los resultados finales en forma de mapa o reportes, tienen aplicabilidad herramientas como los SIG, que permiten centralizar la información, modelarla y optimizar el geoprocesamiento de la misma.

En trabajos futuros se complementará este trabajo con nuevos análisis que permiten la evaluación de riesgos por deslizamientos y la inclusión de una mayor cantidad de datos de lluvia, la modelación del flujo de agua en el interior de la masa de suelo, y que consideren sismos con periodos de recurrencia diferentes ya que este trabajo se limitó a sismos con períodos de retorno de 475 años.

\section{Agradecimientos}

Un especial agradecimiento extiendo al doctor César Augusto Hidalgo y a la doctora Nora Cristina Sabbione, quienes fueron los directores del trabajo de maestría que condujo a los resultados presentados en este artículo, y que con sus valiosos aportes contribuyeron con el desarrollo del mismo. Igualmente, a la Oficina de Catastro Municipal de la ciudad de Medellín por su cooperación con el suministro de información.

\section{Bibliografía}

Área Metropolitana del Valle de Aburrá (2007). Microzonificación y evaluación del Riesgo Sísmico del Valle de Aburrá, publicación institucional, núm. 29, 2007, $184 \mathrm{pp}$.

(2009). "Proyecto en conjunto con los municipios de Medellín y Envigado, Corantioquia y la Universidad Nacional de Colombia. Amenaza, vulnerabilidad y riesgo por movimientos en masa, avenidas torrenciales e inundaciones en el Valle de Aburrá. Formulación de propuestas de gestión”, Libro II, julio, $225 \mathrm{pp}$.

Aristizábal, E. y Gómez, J. (2007). "Inventario de emergencias y desastres en el Valle de Aburrá. Originados por fenómenos naturales y antrópicos en el periodo 1880-2007”, Gestión y Ambiente, Medellín, vol. 10, núm. 2, 2007, pp. 17-30. Asociación Colombiana de Ingeniería Sísmica - AIS (2010). Normas Colombianas de Diseño y Construcción Sismo Resistente, NSR-10, Bogotá, Colombia, 2010.

Baecher, G.B. y Christian J.T. (2003). Reliability and Statistics in Geotechnical Engineering, John Wiley \& Sons., England, 2003, 620 pp. 
Botero, V. (2009). Geo-information for measuring vulnerability to earthquakes: a fitness for use approach, Doctoral Dissertation, Netherlands, International Institute for Geo-information Science and Earth Observation, University of Utrech.

Campos, A.; Holm-Nielsen, N.; Díaz, C.; Rubiano, D.; Costa, C.; Ramirez, F. y Dickson, E. (2012). Análisis de la gestión del riesgo de desastres en Colombia. Un aporte para la construcción de políticas públicas, Banco Mundial, Bogotá, Colombia, 436 pp.

Chowdhury, R.; Flentje, P. and Bhattacharya, G. (2010). Geotechnical Slope Analysis, Taylor \& Francis, London, England, 737 pp.

Christian, J.T.; Ladd, C.C. y Baecher, G.B. (1994). "Reliability Applied to Slope Stability Analysis", Journal of Geotechnical Engineering, ASCE, vol. 120, no. 12, pp. 2180-2207.

Corominas, J.; Van Westen, C.; Frattini, P.; Cascini, L.; Malet, J.; Fotopoulou, S. and Smith, J. (2013). "Recommendations for the quantitative analysis of landslide risk", Bull Eng Geol Environ, pp. 1-55.

Frigerio, S. and Van Westen, C. (2010). "Risk City and Web Risk City: Data Collection, Display, and Dissemination in a Multi-Risk Training Package", Cartography and Geographic Information Science, vol. 37, núm. 2, pp. 119-135.

Hidalgo, C.A.; Vega, J.A.; Assis, A. (2012). "Estimación de amenaza por deslizamiento en proyectos lineales: carreteras en suelos residuales", Memorias del IV Simposio Panamericano de deslizamientos IVSPD, Paipa, Colombia, 2012.

Hidalgo, C.A. (2013). Incertezas, Vulnerabilidade e Avaliação de Risco Devido a Deslizamento em Estradas, tesis de doctorado Departamento de Engeniería Civil y Ambiental, Universidad de Brasilia, Brasil, 250 pp.

Isaza, P.; Martínez, H. e Hidalgo, C. (2016). "Methodology for quantitative landslide risk analysis in residential projects", Habitat International, no. 53, pp. 403-412.

Lan, H.X.; Zhou, C.H.; Wang, L.J.; Zhang, H.Y. and Li, R.H. (2004). "Landslide hazard spatial analysis and prediction using GIS in the Xiaojiang watershed, Yunnan, China", Engineering Geology, no. 76, pp. 109-128.

Martha, T.R.; Van Westen, C.J.; Kerle, N.; Jetten, V. and Vinod Kumar, K. (2013). "Landslide hazard and risk assessment using semi-automatically created landslide inventories", Geomorphology, no. 184, pp. 139-150.

Moreno, H.A.; Vélez, M.A. y Montoya J.D. (2006). "La lluvia y los deslizamientos de tierra en Antioquia: Análisis de su ocurrencia en las escalas interanual, intranual, y diaria", Revista EIA, num. 5, junio, Medellín, pp. 59-69.

Newmark N. (1965). "Effects of earthquakes on dams and embankments", Géotechnique, 15.2, pp. 139-159.

Panahi, M.; Damavandi, A.; Rezaiee, F. and Panahi, S. (2011). "GIS-based earthquake vulnerability of schools in district one of Tehran Municipality using Ana- 
lytical Hierarchy Process", Proceedings of the international annual conference on Geo-information for Disaster Management (Gi4DM).

Promper, C.; Puissant, A.; Malet, J.P. and Glade, T. (2014). "Analysis of land cover changes in the past and the future as contribution to landslide risk scenarios", Applied Geography, no. 53, pp. 11-19.

Remondo, J.; Bonachea, J.; and Cendrero, A. (2008). "Quantitative landslide risk assessment and mapping on the basis of recent occurrences", Geomorphology no. 94 , pp. 496-507.

Shahabi, H. and Hashim, M. (2015). "Landslide susceptibility mapping using GISbased statistical models and Remote sensing data in tropical environment", Scientific Reports 5, Article number 9899.

Torkashvand, A.M.; Irani, A. and Sorur, J. (2014). "The preparation of landslide map by Landslide Numerical Risk Factor (LNRF) model and Geographic Information System (GIS)", The Egyptian Journal of Remote Sensing and Space Sciences, no. 17, pp. 159-170.

Van Westen, C.J.; Castellanos, E., and Kuriakose, S.L. (2008). "Spatial data for landslide susceptibility, hazard, and vulnerability assessment: An overview", Engineering Geology, no. 102, pp. 112-131.

Van Westen, C. (2013). "Remote Sensing and GIS for Natural Hazards Assessment and Disaster Risk Management. Reference Module in Earth Systems and Environmental Sciences", Treatise on Geomorphology, no. 3, pp. 259-298.

Zêrere, J.; García, R.; Oliveira, S. and Reis, E. (2008). "Probabilistic landslide risk analysis considering direct costs in the area north of Lisbon (Portugal)", Geomorphology, no. 94, pp. 467-495. 